\title{
Perspective for development a source of highly polarized ${ }^{3} \mathrm{He}$ - ions
}

\author{
Vadim Dudnikov ${ }^{\mathbf{1}}$ \\ Muons, Inc. \\ 552 Batavia Ave., Batavia IL, USA \\ E-mail: Vadimemuonsinc.com
}

\section{A. Dudnikov}

\section{BINP SBRAS}

11 Lavrentieva pr. Novosibirsk 630090, Russia

E-mail: andreidud@gmail.com

High beam polarization is essential to the scientific productivity of a collider. Polarized ${ }^{3} \mathrm{He}$ ions are an essential part of the nuclear physics programs at existing and future ion-ion and electron-ion colliders such as BNL's RHIC and eRHIC and JLab's ELIC. Ion sources with performance exceeding those achieved today are a key requirement for the development of these next generation high-luminosity highpolarization colliders. A new version of a polarized ${ }^{3} \mathrm{He}^{-}$ion source is needed.

In this article it is proposed a polarized ${ }^{3} \mathrm{He}^{-}$ion source based on the large difference of the autodetachment lifetimes of the different ${ }^{3} \mathrm{He}^{-}$ion hyperfine states. The highest momentum state of 5/3 has the largest lifetime of $\tau \sim 350 \mu$ s while the lower momentum states have lifetimes of $\tau \sim 10 \mu$ s. By producing a ${ }^{3} \mathrm{He}^{-}$ion beam composed of only the $|5 / 2, \pm 5 / 2\rangle$ hyperfine states and then quenching one of the states by an RF resonant field, ${ }^{3} \mathrm{He}^{-}$beam polarization of $95 \%$ can be achieved. A high-brightness arcdischarge ion-source can produce ${ }^{3} \mathrm{He}^{-}$beams with $\sim 1 \mathrm{~mA}$ current with $\sim 90 \%$ polarization.

An integrated ${ }^{3} \mathrm{He}^{-}$ion source design providing high beam polarization could be prepared based on existing BNL equipment that incorporates new designs of the 1) arc discharge plasma generator, 2) extraction system, 3) charge exchange jet, and 4) optimized magnetic separation system. The formation, extraction, and transport of the ion beams in the new source should be computer simulated. Manufacturing techniques should be explored using new materials and fabrication costs should be evaluated. The advanced ${ }^{3} \mathrm{He}^{-}$ion source can be built and tested on the BNL Test Stand for further use in the ion-ion RHIC and ion-electron colliders.

1

Speaker 


POS PROCEEDINGS

XVth International Workshop on Polarized Sources, Targets, and Polarimetry September 9-13, 2013

Charlottesville, Virginia, USA 


\section{Introduction}

The parameters of the Electron-Ion Collider projects that are being actively developed by BNL and JLab are discussed in Ref. [1]. Advanced spin control techniques used in these projects should provide very good polarization preservation including ${ }^{3} \mathrm{He}^{-}$polarization. This means that the final beam polarization after acceleration will be determined by the beam polarization extracted from the ion source, which must be made as high as possible. Polarized ${ }^{3} \mathrm{He}$ ions are particularly important for efficient electron-ion collider operation.

The evolution of polarized ion sources has been presented recently by W. Haeberli [2]. Review of polarized ${ }^{3} \mathrm{He}$ ions beam production has been presented in Ref. [3]. Old ion sources have polarized ${ }^{3} \mathrm{He}$ ion beam intensity of $\mathrm{nA}$ scale. Since the efficiency of experiments is proportional to the square of the polarization, $\mathrm{P}^{2}$, having the highest possible degree of polarization is very important. For polarized ${ }^{3} \mathrm{He}^{++}$production, it was proposed to use ionization of nuclear polarized ${ }^{3} \mathrm{He}^{0} \uparrow$ by electrons in an electron beam ion source (EBIS) [3,4]:

$$
{ }^{3} \mathrm{He}^{0} \uparrow+\mathrm{e} \Rightarrow{ }^{3} \mathbf{H e}^{++} \uparrow+3 \mathrm{e}
$$

The expected beam intensity is about $2.5 \cdot 10^{11}{ }^{3} \mathrm{He}^{++} /$pulse with nuclear polarization $\mathrm{P}>70 \%$.

For polarized ${ }^{3} \mathrm{He}^{++}$production, one can use also the same high-current arc-discharge source (developed in BINP [5] and used in the BNL OPPIS upgrading [6]) with pulsed injection of nuclear polarized ${ }^{3} \mathrm{He}^{0} \uparrow$ atoms (polarized by optical pumping) into an arc- discharge plasma source [7]. For protection of the nuclear polarization during step-by-step ionization a strong magnetic field can be used.

Another proposed option is to use resonant charge exchange ionization of polarized ${ }^{3} \mathrm{He}^{0} \uparrow$ in a storage tube by an incident ${ }^{4} \mathrm{He}^{+},{ }^{4} \mathrm{He}^{++}$plasma jet as shown in Fig. 1 from [8].

$$
{ }^{3} \mathrm{He}^{0} \uparrow+{ }^{4} \mathrm{He}^{++} \Rightarrow{ }^{3} \mathbf{H e}^{++} \uparrow+{ }^{4} \mathrm{He}^{0}
$$

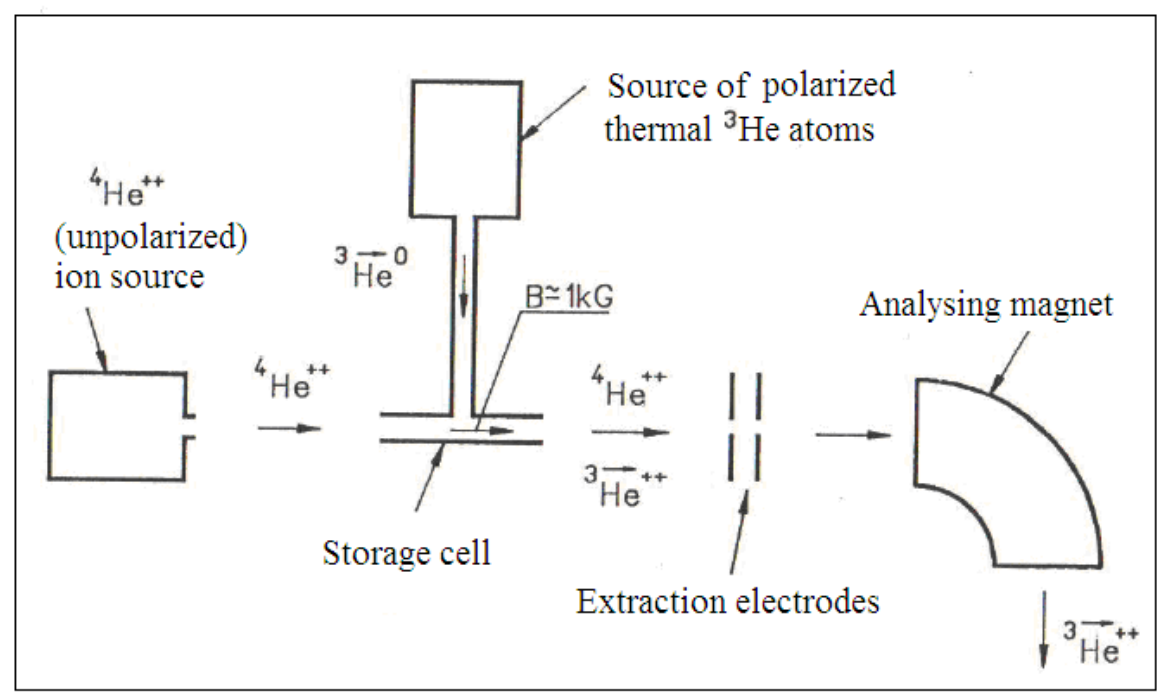

Figure 1: Schematic view of a polarized ${ }^{3} \mathrm{He}$ ion source [8]. The BINP arc discharge source can be used as the source of ${ }^{4} \mathrm{He}^{++}[5]$. 
The proposed methods of polarized $3 \mathrm{He}$ ion production were discussed but were never tested.

\subsection{Perspectives for production of polarized ${ }^{3} \mathrm{He}^{-}$ions}

Polarized ${ }^{3} \mathrm{He}^{-}$ions could be produced using the high-brightness arc-discharge ion source with geometrical focusing and low gas consumption developed at BINP and used for BNL OPPIS upgrading [9]. With 2A of ${ }^{3} \mathrm{He}^{+}$beam current, up to $0.1 \mathrm{~A} \mathrm{of}{ }^{3} \mathrm{He}^{-}$beam can be produced by charge exchange in an alkali vapor target and up to $2 \mathrm{~mA}$ of highly polarized ${ }^{3} \mathrm{He}^{-}$ions can be produced [5,7]. With a pulsed gas valve [10] it is possible to have a low gas consumption, which is important, because ${ }^{3} \mathrm{He}$ gas is very expensive.

The basic idea of this proposal can be traced back to the alpha particle diagnostics that are being developed for the ITER project in France. For this purpose, a $1 \mathrm{MeV}, 10 \mathrm{~mA} \mathrm{He}$ ion source is under development $\left(\mathrm{He}^{+}\right.$current should be $\sim 3 \mathrm{~A}$ with low emittance), [11]. Electron autodetachment from metastable $\mathrm{He}$ - ions is used for production of the fast ground-state $\mathrm{He}^{0}$. Metastable $\mathrm{He}^{-}$has three different lifetimes of $\sim 10 \mu \mathrm{s}, \sim 16 \mu \mathrm{s}$ and $\sim 350 \mu \mathrm{s}$.

We started by looking for lifetime differences of the different hyperfine states, then as described earlier it is possible to use these differences for polarized ${ }^{3} \mathrm{He}^{-}$production. It was found that these differences did exist, and thus polarized ${ }^{3} \mathrm{He}^{-}$production is possible.

Theoretical estimation of the autodetacment lifetimes for the different states of $\mathrm{He}^{-}$ions was presented in Ref. [12].

It is not possible to attach an electron to the a helium atom in the ground state to form a stable negative ion, but an electron can be attached to the helium atom in the $1 \mathrm{~s} 2 \mathrm{~s} 3 \mathrm{~S}$ excited state of the helium atom leading to the formation of a metastable $\mathrm{He}^{-}\left(1 \mathrm{~s} 2 \mathrm{~s} 2 \mathrm{p} 4 \mathrm{P}_{\mathrm{J}}\right)$ ion, which has different autodetachment lifetimes. The three $4 \mathrm{P}_{\mathrm{J}}$ levels are forbidden to decay via the Coulomb interaction, the $4 \mathrm{P}_{3 / 2 ; 1 / 2}$ levels can decay by spin-orbit and spin-spin interaction, whereas the $4 \mathrm{P}_{5 / 2}$ level only decays by spin-spin interaction. The fine structure of a helium negative ion was resolved in Ref. [13]. With a lifetime exceeding $350 \mu \mathrm{s}$, the $\mathrm{He}^{-}\left(1 \mathrm{~s} 2 \mathrm{~s} 2 \mathrm{p} 4 \mathrm{P}_{5 / 2}\right)$ ion become an attractive object to study. Storage rings [14] and ion trap experiments [15] were used for accurate measurements of the lifetimes of the metastable $\mathrm{He}^{-}$ions.

The produced data can be used for development of a polarized ${ }^{3} \mathrm{He}^{-}$ion source based on the large difference in the autodetachment lifetimes of the different ${ }^{3} \mathrm{He}^{-}$ion hyperfine states. The highest momentum state of $5 / 3$ has the largest lifetime of $\sim 350 \mu$ s while the lower momentum states have lifetimes of $\sim 10 \mu$ s. By producing a ${ }^{3} \mathrm{He}^{-}$ion beam composed of only the $|5 / 2, \pm 5 / 2\rangle$ hyperfine states and then quenching one of the states by an RF resonant field, ${ }^{3} \mathrm{He}^{-}$beam polarization of $95 \%$ can be achieved. Being one of the 24 equally populated hyperfine states, a polarized ${ }^{3} \mathrm{He}^{-}$beam has $\sim 4 \%$ intensity of all ${ }^{3} \mathrm{He}^{-}$beam, which in turn can have up to $5 \%$ of a ${ }^{3} \mathrm{He}^{+}$beam intensity. The high-brightness arc-discharge ion source with geometrical focusing and low gas consumption developed in BINP and used for BNL OPPIS upgrading [5,6,9] can be used for production of ${ }^{3} \mathrm{He}^{-}$beams with $\sim 1 \mathrm{~mA}$ current and $\sim 90 \%$ polarization.

An analogous method of polarized ${ }^{3} \mathrm{He}^{-}$ions production was proposed in [16] in 1964. However, the intensity of the $\mathrm{He}^{+}$ion beam was very low at that time and the lifetimes of $\mathrm{He}-$ ions were unknown. With a very small efficiency of the ${ }^{3} \mathrm{He}^{+}$transformation to the polarized 
${ }^{3} \mathrm{He}^{-}$, there was no hope for any useful beam production and this method was not considered and tested.

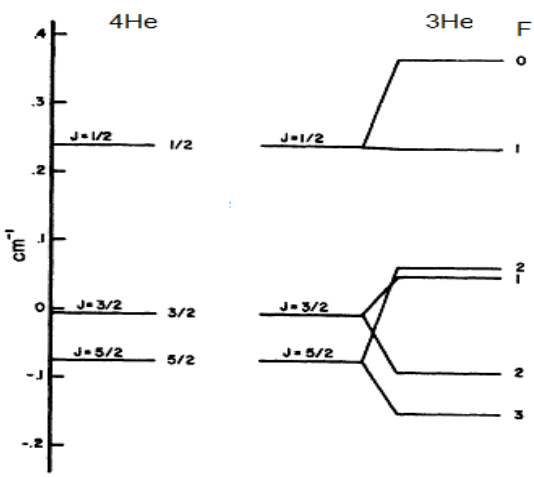

Figure 2: Calculated fine and hyperfine structure of (1s, 2s, 2p) 4P states in ${ }^{4} \mathrm{He}^{-}$and ${ }^{3} \mathrm{He}^{-}$[12].

The calculated fine and hyperfine structure of the (1s, 2s, 2P) 4P states in ${ }^{3} \mathrm{He}^{-}$and ${ }^{4} \mathrm{He}^{-}$are shown in Fig.2. The calculated lifetimes of the (1s, 2s, 2P) ${ }^{4} \mathrm{P}^{-}$states of ${ }^{3} \mathrm{He}^{-}$and ${ }^{4} \mathrm{He}^{-}$are shown in Table III from [12]. While the calculations are not quantitively correct, they demonstrate the main feature of ${ }^{3} \mathrm{He}^{-}$ions: the higher the state's momentum the longer its lifetime.

TABLE III. Calculated lifetimes in $\mu \mathrm{sec}$ of the states arising from $(1 s, 2 s, 2 p){ }^{4} P$ in $\mathrm{He}^{3}, \mathrm{He}^{4}, \mathrm{Li}^{6}$, and $\mathrm{Li}^{7}$, including the effects of the $(1 s, 2 s, 2 p)^{2} P$ levels. Those lifetimes marked by asterisks involved large cancellations and must be considered extremely unreliable.

\begin{tabular}{|c|c|c|c|c|c|c|c|}
\hline \multicolumn{2}{|c|}{$\mathrm{He}^{3}$} & \multicolumn{2}{|c|}{$\mathrm{He}^{4}$} & \multicolumn{2}{|c|}{$\mathrm{Li}^{6}$} & \multicolumn{2}{|c|}{$\mathbf{L i}^{7}$} \\
\hline$|3,5 / 2\rangle$ & 1000 & $|5 / 2,5 / 2\rangle$ & 1000 & $17 / 2,5 / 2>$ & 5. 88 & $|4,5 / 2\rangle$ & 5.88 \\
\hline $12,5 / 2>$ & 370 & $|3 / 2,3 / 2\rangle$ & 33 & $|5 / 2,5 / 2\rangle$ & 5.88 & $13,5 / 2\rangle$ & 1.47 \\
\hline$|2,3 / 2\rangle$ & 65 & $|1 / 2,1 / 2\rangle$ & $3500^{*}$ & $|3 / 2,5 / 2\rangle$ & 5.05 & $|2,5 / 2\rangle$ & 2.30 \\
\hline$|1,3 / 2\rangle$ & 29 & & & $15 / 2,3 / 2>$ & 0.24 & $|1,5 / 2\rangle$ & 5.87 \\
\hline$|1,1 / 2\rangle$ & 21 & & & $|3 / 2,3 / 2\rangle$ & 0.25 & $13,3 / 2\rangle$ & 0.28 \\
\hline \multirow[t]{5}{*}{$|0,1 / 2\rangle$} & 80 & & & $|1 / 2,3 / 2\rangle$ & 0.29 & $|2,3 / 2\rangle$ & 0.30 \\
\hline & & & & $|3 / 2,1 / 2\rangle$ & $100^{*}$ & $|1,3 / 2\rangle$ & 0.32 \\
\hline & & & & $|1 / 2,1 / 2\rangle$ & $29^{*}$ & $10,3 / 2\rangle$ & 0.35 \\
\hline & & & & & & $|2,1 / 2\rangle$ & $20.0^{*}$ \\
\hline & & & & & & $|1,1 / 2\rangle$ & $10.2^{*}$ \\
\hline
\end{tabular}

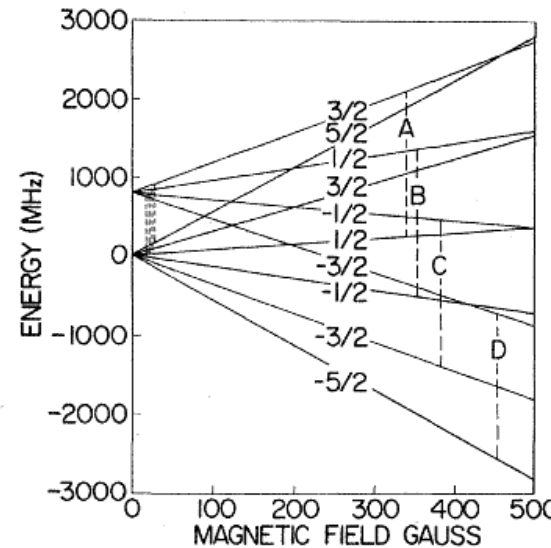

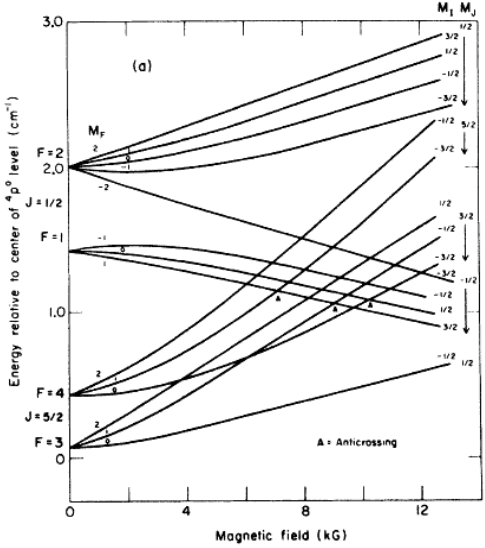

$\mathrm{b}$

Figure 3: a) Energy sublevels of ${ }^{4} \mathrm{He}^{-}$as a function of magnetic field for the $\mathrm{J}=5 / 2$ and $3 / 2$ levels. The $\mathrm{J}=1 / 2$ level lies above these two with a zero-field energy of $8663 \mathrm{MHz}$ [13]. 
b) Energy as a function of magnetic field for various ( $1 \mathrm{~s} 2 \mathrm{~s} 2 \mathrm{p}) 4 \mathrm{P}$ substates of ${ }^{7} \mathrm{Li}$. The positions of the three observed anticrossings are indicated by A [17].

Shifts of the fine structure levels of ${ }^{4} \mathrm{He}^{-}$in magnetic field and their energies (frequencies) are shown in Fig. 3a from [13]. Correct experimental lifetimes were presented in [14,15]: $10 \mu$ s for $\mathrm{j}=1 / 2,16 \mu$ s for $\mathrm{j}=3 / 2$ and $\sim 350 \mu$ s for $\mathrm{j}=5 / 2$.

For fine structure measurements, a $\mathrm{He}^{-}$beam produced from $\mathrm{He}^{+}$by double charge exchange in potassium vapor is directed into a drift tube, which contains an RF interaction region and a Faraday-cup ion detector. A population difference evolves between the states both before they enter the RF region and after they leave it. When the $J=-5 / 2$ and $-1 / 2$ states are coupled by the RF field, there is a decrease in the detected ion current [13]. The transition frequencies between fine-structure components are:

$$
\begin{gathered}
\Delta_{53}=825.23+0.82 \mathrm{MHz}, \\
\Delta_{51}=8663+56 \mathrm{MHz} .
\end{gathered}
$$

The levels energy ordering of the levels shown here is that found theoretically by Manson, while the numerical values are those measured experimentally. The transitions, which have been observed, are indicated by the vertical dashed lines in Fig. 3a from [13].

Determination of the energies and lifetimes of the metastable auto-ionizing (1s2s2p) P states of ${ }^{6} \mathrm{Li}$ and ${ }^{7} \mathrm{Li}$ (analogs of ${ }^{3} \mathrm{He}^{-}$levels) by the Zeeman-Quenching Technique was described in [17]. The positions of the three observed anticrossings are indicated by A in Fig. $3 b$.

\subsection{Proposal for experimental testing of $\mathrm{He}^{-}$production.}

Using the arc discharge source (from BINP as used in OPPIS $[5,6,9]$ ) one can extract up to $\mathrm{I}+\sim 2 \mathrm{~A}$ of $\mathrm{He}^{+}$at $6-12 \mathrm{keV}$ energy with good emittance and up to $\sim 0.1 \mathrm{~A}$ of $\mathrm{He}^{-}$can be obtained

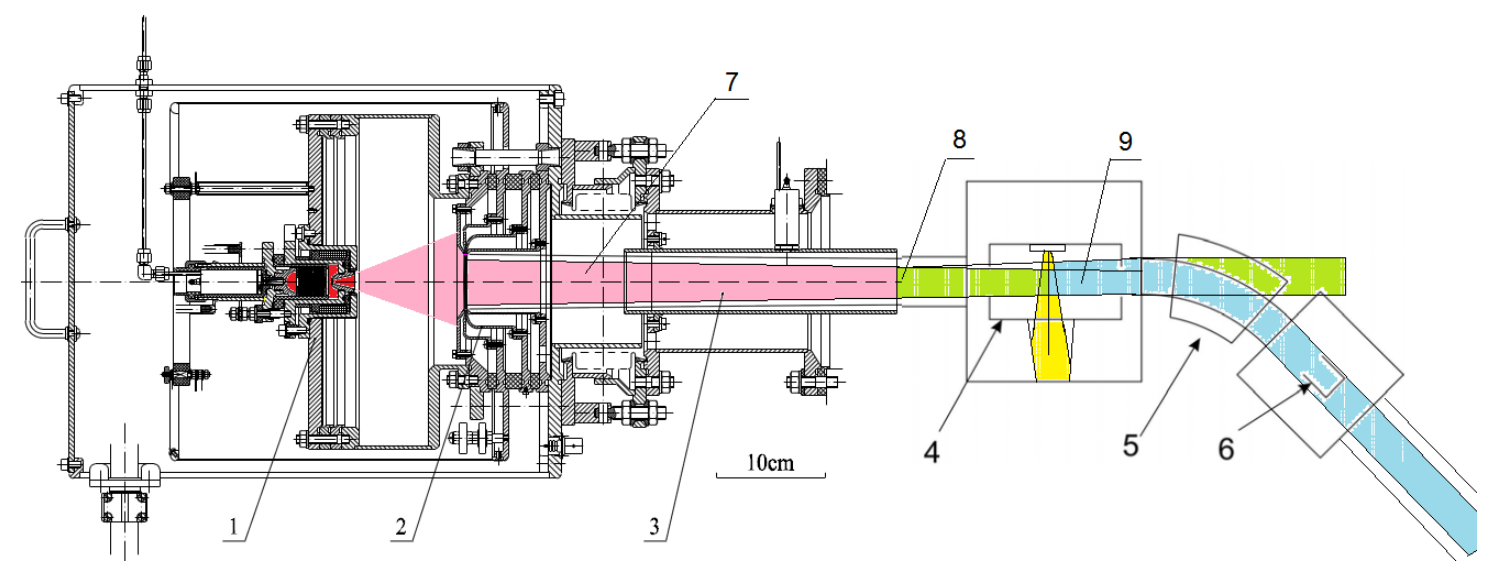

Figure 4: A schematic of an experiment on $\mathrm{He}^{-}$beam production. 1- $\mathrm{He}^{+}$source, 2 - extraction system, 3 - space charge compensation, $4-\mathrm{Cs}(\mathrm{Rb}, \mathrm{K})$ jet target, 5 - bending magnet, 6 - decay channel with solenoid and RF transition, 7-He+ beam; 8-space charge compensated beam; $9-\mathrm{He}$ beam.

by charge exchange in a Potassium jet target. After some time of flight $(\sim 30 \mu \mathrm{s}, \sim 30 \mathrm{~m})$ in a magnetic field, the components with momentum projections $1 / 2$ and $3 / 2$ should be autoionized 
(up to $95 \%$ ), leaving only ${ }^{3} \mathrm{He}^{-}$ions with components [5/2,+-5/2>]. Than using RF to induce a transition of one of the components to the zero state, one can produce a ${ }^{3} \mathrm{He}^{-}$beam with nuclear polarization close to $\sim 95 \%$.

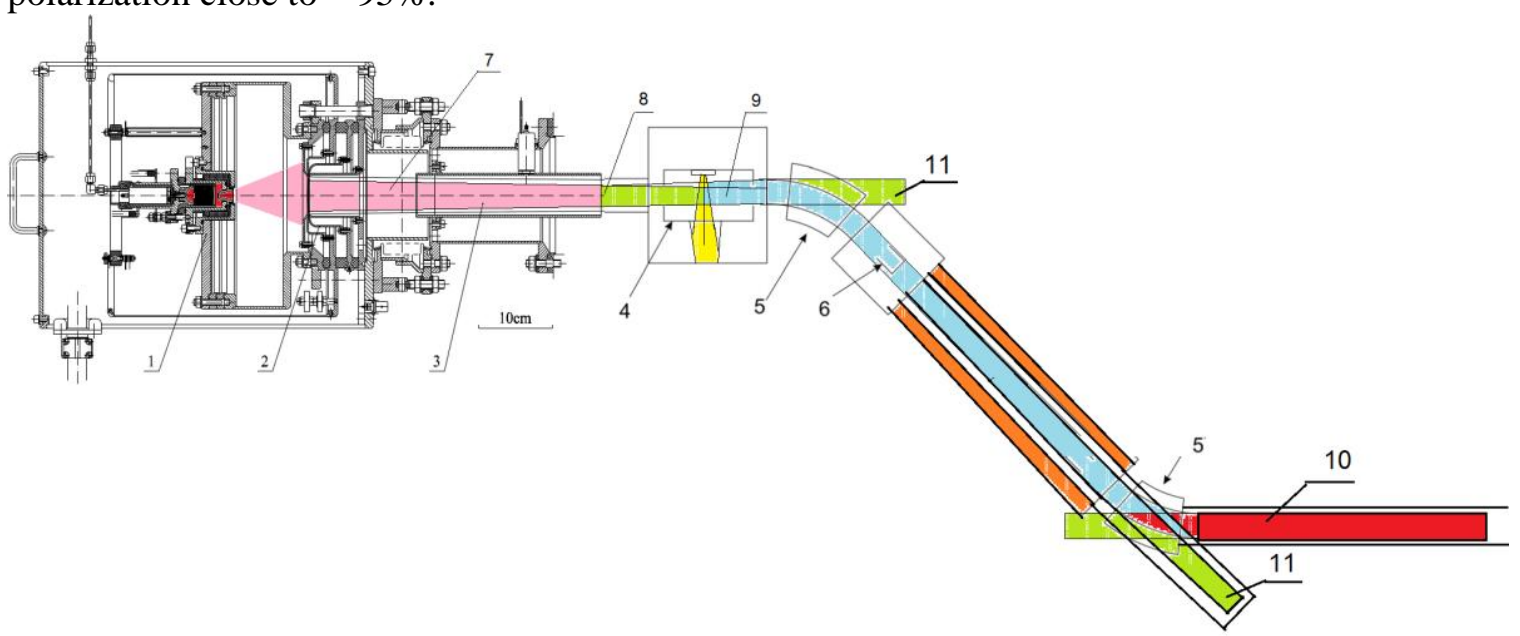

Figure 5: A schematic of the $3 \mathrm{He}$ - ion source. 1- arc discharge He+ source, 2 - extraction system, 3 - space charge compensation, 4 - Cs (Rb, K) target, 5 - bending magnet, 6 - Decay channel with solenoid and RF transition, 7- $\mathrm{He}^{+}$beam; 8 -space charge compensated beam; 9- $\mathrm{He}^{-}$ beam; 10-polarized ${ }^{3} \mathrm{He}^{-}$beam; $11-{ }^{3} \mathrm{He}$ neutral beam.

A schematic of an experiment using BNL equipment to measure He- beam production is shown in Fig. 4. A high brightness beam of $\mathrm{He}^{+}$ions (7) beam is generated by an arc-discharge plasma source (1) and is formed by a multigrid focusing extraction system (2). A pulsed gas target (3) is used for space charge compensation. A vapor jet target (4) (K, Rb or Cs) can be used for $\mathrm{He}^{+}$to $\mathrm{He}^{-}$beam (9) conversion.

Short lived $\mathrm{He}^{-}$ions can eject electrons during their flight in the decay channel (6) with solenoid and RF transition producing a polarized ${ }^{3} \mathrm{He}^{-}$beam (10) as shown in Fig. 5. To prevent intrabeam stripping, the ${ }^{3} \mathrm{He}^{-}$beam is separated from the intense $\mathrm{He}^{+}$and $\mathrm{He}^{0}$ beams by the bending magnet (5). The beam pipes must be cooled below $150 \mathrm{~K}$ to prevent $\mathrm{He}^{-}$stripping by the black body radiation.

A schematic of an experiment on $\mathrm{He}^{-}$beam production is shown in Figs. 4 and 5. An arc discharge ion source can be used for generation of high-brightness $\mathrm{He}^{+}$beams with intensity up to $3 \mathrm{~A}$ at energy $\sim 10-15 \mathrm{keV}$. A pulsed Xe gas target can be used for space charge compensation and production of metastable $\mathrm{He}^{*}$. A second $(\mathrm{K})$ vapor jet target can be used for $\mathrm{He}^{-}$production.

A schematic of energy diagrams of the ground state and the lowest excited states of ${ }^{4} \mathrm{He}$, and the 1s2s 2 p 4Po state of ${ }^{4} \mathrm{He}^{-}$are shown in Fig. 6 from [14].

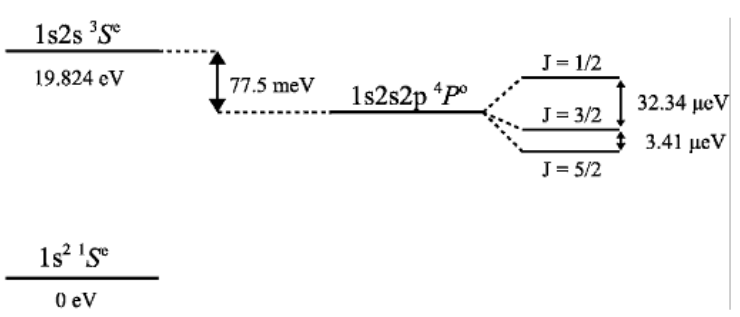

FIG. 6. Schematic energy diagrams of the ground state and the lowest excited state of ${ }^{4} \mathrm{He}$, and the 1 s2s $2 p$ 4Po state of ${ }^{4} \mathrm{He}^{-}[14]$. 
The ${ }^{4} \mathrm{He}^{-}$ions were produced in double collisions $\left({ }^{4} \mathrm{He}^{+}+\mathrm{Cs}--{ }^{4} \mathrm{He}^{*},{ }^{4} \mathrm{He}^{*}+\mathrm{Cs}---{ }^{4} \mathrm{He}^{-}\right)$ of $2.5 \mathrm{keV}^{4} \mathrm{He}^{+}$in a cesium charge exchange cell.

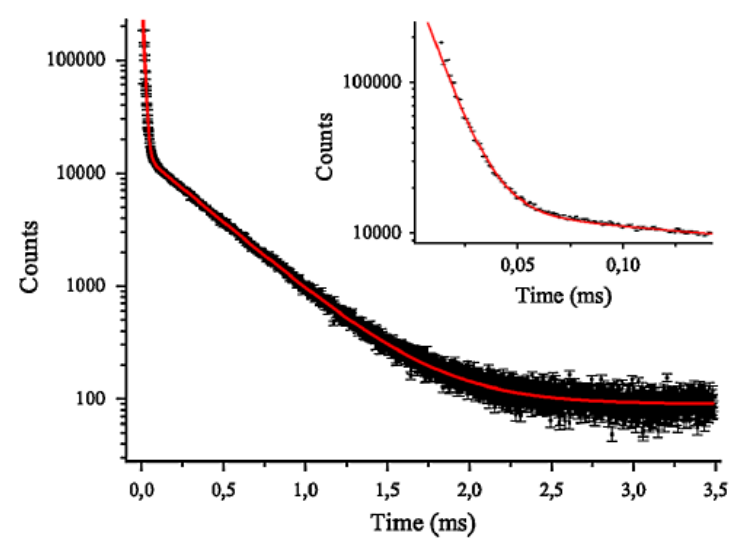

a

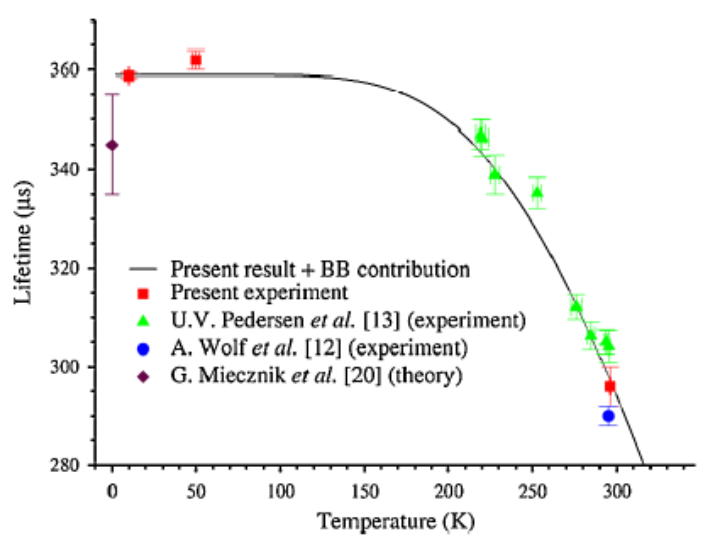

b

Figure 7: a) Decay curve of ${ }^{4} \mathrm{He}^{-}$measured at $10 \mathrm{~K}$. The solid curve is a fit to the data. The inset shows the time region in which the decay of the short lived $\mathrm{J}=1 / 2$ and $\mathrm{J}=3 / 2$ levels dominate the intensity [15]; b) Temperature dependence of the measured lifetime of the $1 \mathrm{~s} 2 \mathrm{~s} 2 \mathrm{p} 4 \mathrm{P}^{\mathrm{o}}{ }_{5 / 2}$ level of ${ }^{4} \mathrm{He}^{-}$.

The effect on the blackbody radiation on the photodetachment decay rate can be readily seen in Fig. $7 \mathrm{~b}$ as a decrease in the measured lifetime above $100 \mathrm{~K}$ [15].

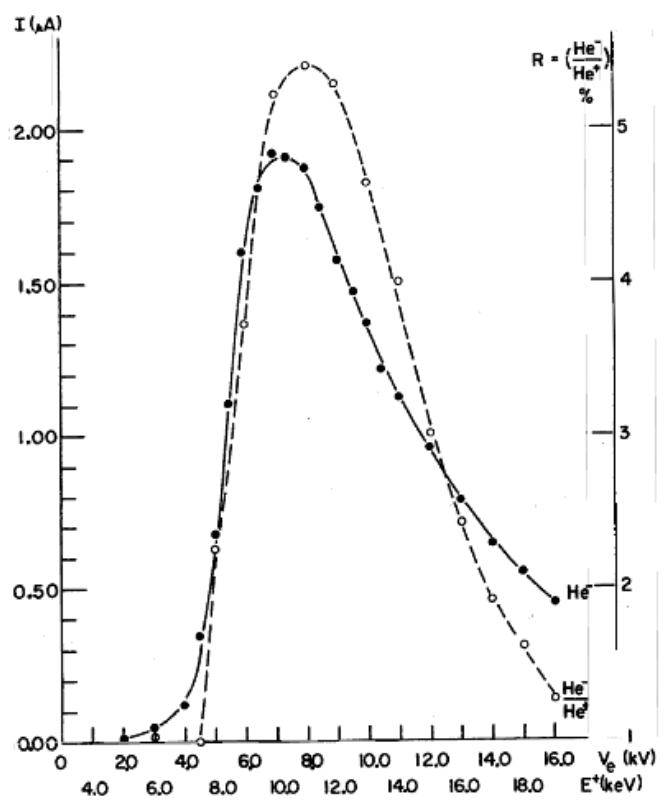

Figure 8: $\mathrm{He}^{-} / \mathrm{He}^{+}$yield and beam intensity vs. $\mathrm{He}^{+}$energy [18].

Fig. 8 shows the $\mathrm{He}^{-} / \mathrm{He}^{+}$yield and beam intensity vs. $\mathrm{He}^{+}$energy with an optimal $\mathrm{K}$ target [18]. More then $5 \%$ of $\mathrm{He}^{+}$ions can be converted into $\mathrm{He}^{-}$ions. With $2 \mathrm{~A} \mathrm{He}^{+}$current from the BINP arc discharge source it is possible to have $\sim 50-100 \mathrm{~mA}$ of $\mathrm{He}^{-}$ions. Up to $\sim 2 \mathrm{~mA}$ of ${ }^{3} \mathrm{He}^{-}$ with high nuclear polarization can be produced. 
For a preliminary feasibility testing of $\mathrm{He}^{-}$ion production, it is possible to use an upgraded BNL OPPIS assembly $[6,9]$ with a low solenoid current. The $\mathrm{He}^{+}$beam can be generated by an arc discharge plasma source and formed by a multigrid extraction system with space charge compensation using a pulsed $\mathrm{Xe}$ target. The $\mathrm{He}^{-}$beam can be generated by charge exchange in the $\mathrm{Rb}$ cell in a weak magnetic field with optical pumping and without optical pumping. Furthermore, other configurations of the BNL OPPIS assembly can be used to study ${ }^{4} \mathrm{He}^{-}$ production in a $\mathrm{Rb}$ cell as well as $\mathrm{He}^{-}$production with a $\mathrm{K}$ jet charge exchange cell.

Assembly of a $\mathrm{He}^{-}$source with a $\mathrm{K}$ jet charge exchange cell for very efficient $\mathrm{He}$ - ion production is also possible with the BNL OPPIS. The first Xe gas target can be used to improve space charge compensation and production of excited fast He* atoms. A bending magnet after the charge exchange cell should be used for separation of $\mathrm{He}^{-}$ion from the $\mathrm{He}^{+}$and $\mathrm{He}^{0}$ beams as shown in Figs. 4 and 5.

The polarized ${ }^{3} \mathrm{He}^{-}$beam can be converted into ${ }^{3} \mathrm{He}^{++}$by stripping after fast acceleration. A small tandem (fabricated by the VSEA division of Applied Material) or a small linac (fabricated by Axcelis Corp.) used for ion implantation can be used for this acceleration. The polarized ${ }^{3} \mathrm{He}^{-}$ can be accelerated in the BNL Tandem that was used for heavy ion injection to the AGS booster for RHIC.

For preliminary feasibility testing of $\mathrm{He}^{-}$ion production it is possible to use an upgraded BNL OPPIS assembly [6] as shown in Fig. 9 with a low current in solenoid (magnetic

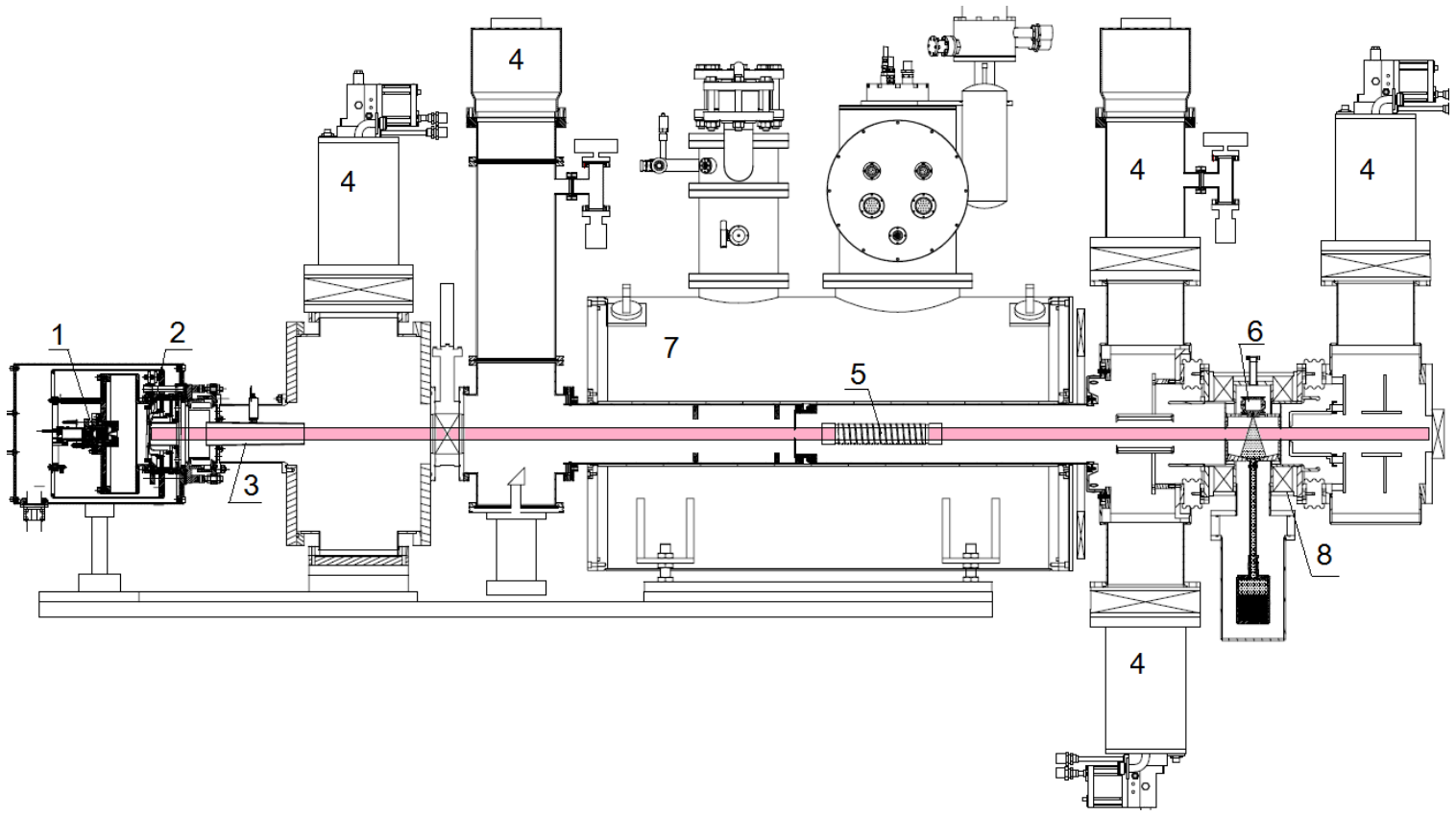

FIG. 9. BNL OPPIS Assembly, which can be used for feasibility test of $\mathrm{He}^{-}$ion production. 1arc discharge plasma source; 2-extraction system; 3-gas target; 4-vacuum pumps; 5- $\mathrm{Rb}(\mathrm{Cs})$ vapor cell; 6-vapor jet target; 7- superconductor solenoid; 8- magnetic coils of Sona transition.

field below $1 \mathrm{kG}$ ). $\mathrm{He}^{+}$beam can be generated by an arc discharge plasma source (1) and formed by multigrig extraction system (2) with a space charge compensation by pulsed Xe target (3). He- beam can be generated by charge exchange in the Rb cell (5) in weak magnetic field with optical pumping and without optical pumping. 
It is interesting to test ${ }^{4} \mathrm{He}^{-}$production in the $\mathrm{Rb}$ cell with and without optical pumping. It is useful also to test $\mathrm{H}^{-}$production in the $\mathrm{Rb}$ cell with and without optical pumping. It is possible to hope that the cross section for $\mathrm{H}^{-}$production by electron an capture from excited $\mathrm{Rb}$ can be larger than from $\mathrm{Rb}$ in the ground state. As second approach it is possible to use an equipment combination shown in Fig. 10 with the arc discharge source moved close to the Rb cell.

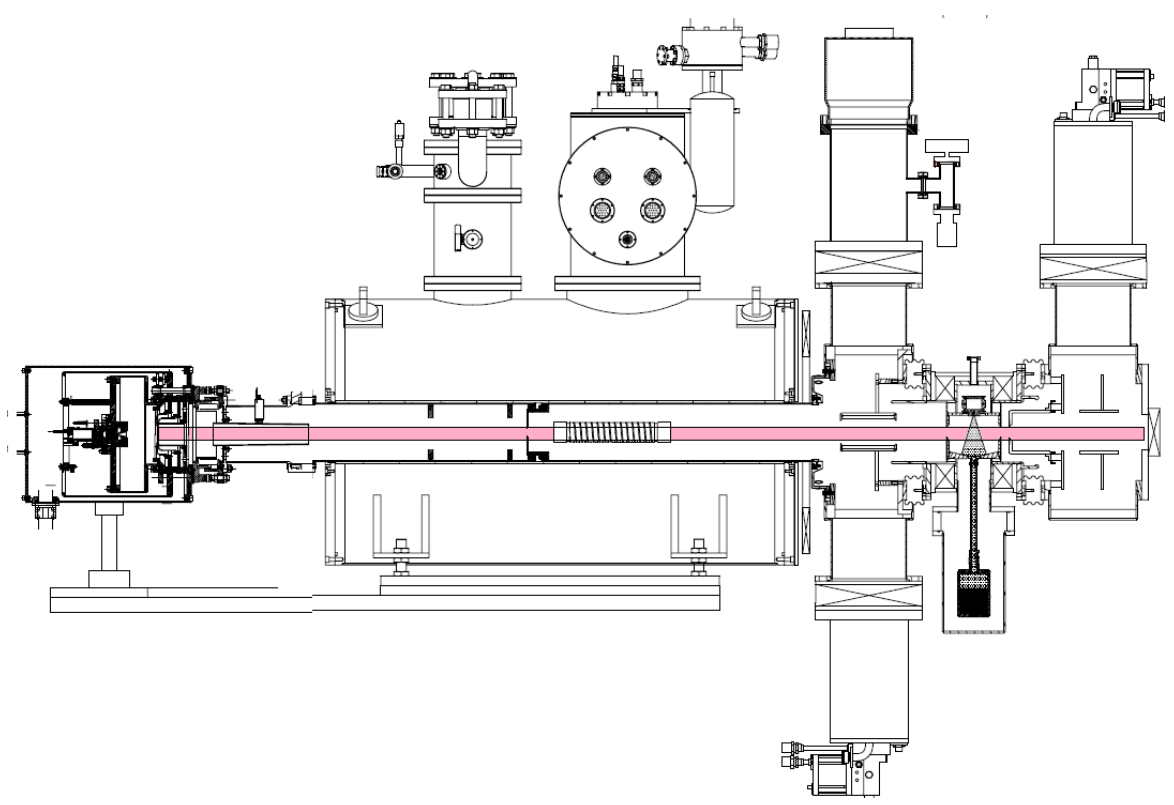

FIG. 10. He source assembly with the arc discharge source located closely to the Rb cell for higher efficiency of $\mathrm{He}^{-}$production.

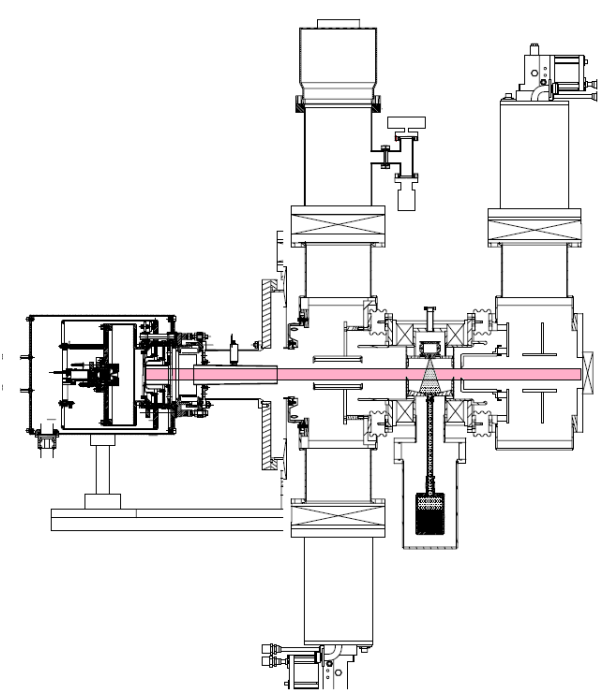

FIG. 11. He- source assembly with a $\mathrm{K}$ jet charge exchange cell for high efficiency $\mathrm{He}^{-}$ion production.

A He ${ }^{-}$source assembling with a Potassium jet charge exchange cell for high efficiency $\mathrm{He}^{-}$ion production is shown in Fig. 11. The first Xe gas target can be used for improvement of space charge compensation and production of excited fast $\mathrm{He}^{*}$ atoms. A bending magnet after the 
charge exchange cell should be used for $\mathrm{He}^{-}$ion separation from $\mathrm{He}^{+}$and $\mathrm{He}^{0}$ beams as shown in Figs. 4 and 5.

\section{References}

[1] V. Morozov, Ion spin dynamics at mEIC, 2013 International Workshop on Polarized Sources, Targets \& Polarimetry (PSTP), Charlottesville in September 2013;

V. Ptitsyn, Electron and ion spin dynamics at eRHIC, 2013 International Workshop on Polarized Sources, Targets \& Polarimetry (PSTP), Charlottesville in September 2013;

A.Kondratenko et al., Preservation And Control Of The Proton And Deuteron Polarizations In The Proposed Electron-Ion Collider at Jefferson Lab, DSPIN-2012, Sep 17- 22, 2012, Dubna.

[2] W. Haeberli, The Toolbox of Proton Spin Physics in Historical Perspective, PSTP-2007, BNL, USA, AIP Conf. Proc. 980 (2008), pp. 3-14.

[3] A.Zelenski, J,Alessi, E.Beebe, A.Pikin, M.Farkhondeh, W.Franklin, A. Kocoloski, R.Milner, C. Tschalaer, E.Tsentalovich, E.Hughes, A proposal for a polarized $3 H^{++}$ion source with the EBIS ionizer for RHIC, SPIN 2004, Trieste, Italy, 2004.

[4] J. Maxwell, Polarized He-3 Source Development for RHIC, 2013 International Workshop on Polarized Sources, Targets \& Polarimetry (PSTP), Charlottesville in September 2013

[5] Bel'chenko Yu. I, Davydenko V. I., Derevyankin G. E., Dimov G. I., Dudnikov V. G., Morozov I. I., Roslyakov G. V., Shabalin A. L. Ion sources at the Novosibirsk Institute of Nuclear Physics, Rev. Sci. Instrum. 61, 378-384 (1990).

[6] A. Zelenski et al., RHIC polarized source upgrade, 2013 International Workshop on Polarized Sources, Targets \& Polarimetry (PSTP), Charlottesville in September 2013.

[7] V. Dudnikov, Perspectives of Highly Polarized Ion Sources Development, 2013 International Workshop on Polarized Sources, Targets \& Polarimetry (PSTP), Charlottesville in September 2013.

[8] A. Belov, Production of Polarized Ions with Nearly Resonant Charge-Exchange Collisions in Plasma, PSTP-2007, BNL, USA, AIP conf. Proc. 980 (2008), pp. 209-220.

[9] A.N. Zelensky, Towards 100\% polarization in the ion source, BNL report, 2005.

[10] G. E. Derevyankin, V. G. Dudnikov, and P. A. Zhuravlev, Electromagnetic shutter for a pulsed gas inlet into vacuum units, Pribory I technika Experimenta, 5, p.168-169 (1975).

[11] M. Kisaki, et al., Development of a strongly focusing high-intensity He-ion source for a confined alpha particle measurement at ITER, Review of Scientific Instruments, 79, 02C113 -(2008).

[12] Steven Trent Manson, Theoretical Considerations for Auto-ionizing States with Microsecond Lifetimes, PHYSICAL REVIEW A, V. 3, N. 1, (1971).

[13] D. I. Mader and R. Novick, Fine Structure of the Helium Negative Ion, Phys. Rev. Lett., 29, 4,199, (1972).

[14] U. V. Pedersen, M. Hyde, S. P. Moller, and T. Andersen, Lifetime measurement of He- utilizing an electrostatic ion storage ring, PHYSICAL REVIEW A, V. 64, 012503, (2001).

[15] P. Reinhed, A. Orba'n, J. Werner, S. Rose'n, R. D. Thomas, I. Kashperka, H. A. B. Johansson, D. Misra, L. Brannholm, M. Bjorkhage, H. Cederquist, and H. T. Schmidt, Precision Lifetime Measurements of He_in a Cryogenic Electrostatic Ion-Beam Trap, PRL 103, 213002 (2009) PHYSICAL REVIEW LETTERS, 2009, 213002.

[16] P. Feldman and B. Novick, Polarization of 3He negative ions, International Congress of Nuclear Physics, Paris, 1964, 785-786; P. Feldman and R. Novick, in Comptes Rendus du Congres International de Physique Nucleaire, Paris, 1964, edited by P. Gugenberger (CNRS, Paris, 1964), Vol. II, 4a/C144, pp. 785-786.

[17] M. Levitt and R. Novick, P. D. Feldman, Determination of Energies and Lifetimes of the Metastable Auto-ionizing (1s2s2p) P States of 6Li and 7Li by a Zeeman-Quenching Technique, PHYSICAL REVIEW A, V. 3, (1971).

[18] A. Szanto de Toledo and O. Salada, Production of Negative Helium ions, Revista Brasileira de Física, Vol. 7, No 1, (1977). 